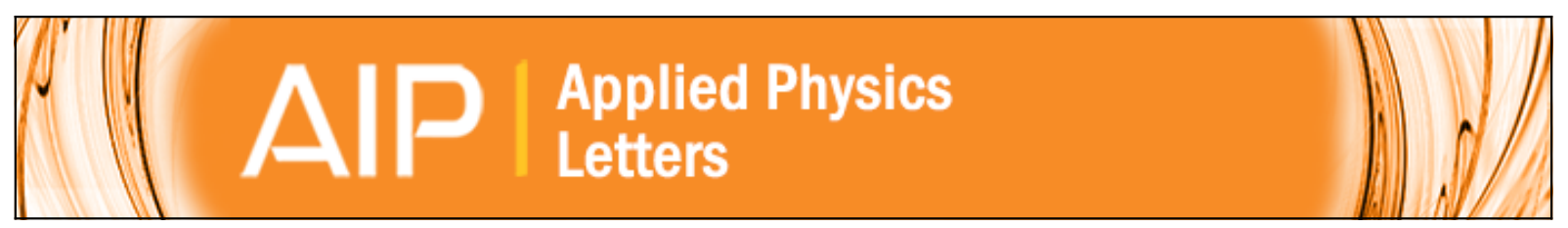

\title{
Self-excited coupled-microcantilevers for mass sensing
}

Daichi Endo, Hiroshi Yabuno, Keiichi Higashino, Yasuyuki Yamamoto, and Sohei Matsumoto

Citation: Applied Physics Letters 106, 223105 (2015); doi: 10.1063/1.4921082

View online: http://dx.doi.org/10.1063/1.4921082

View Table of Contents: http://scitation.aip.org/content/aip/journal/apl/106/22? ver=pdfcov

Published by the AIP Publishing

\section{Articles you may be interested in}

Self-excited coupled cantilevers for mass sensing in viscous measurement environments

Appl. Phys. Lett. 103, 063104 (2013); 10.1063/1.4817979

Bianalyte mass detection with a single resonant microcantilever

Appl. Phys. Lett. 94, 011901 (2009); 10.1063/1.3050460

Resonance-mode effect on microcantilever mass-sensing performance in air Rev. Sci. Instrum. 79, 074301 (2008); 10.1063/1.2949390

Self-exciting, self-sensing $\mathrm{Pb} \mathrm{Zr} 0.53 \mathrm{Ti} 0.47 \mathrm{O} 3 / \mathrm{Si} \mathrm{O} 2$ piezoelectric microcantilevers with femtogram/Hertz sensitivity

Appl. Phys. Lett. 89, 023506 (2006); 10.1063/1.2219994

Ultrasensitive mass sensing using mode localization in coupled microcantilevers

Appl. Phys. Lett. 88, 254102 (2006); 10.1063/1.2216889

\section{AIP $\mid$ APL Photonics}

APL Photonics is pleased to announce Benjamin Eggleton as its Editor-in-Chief

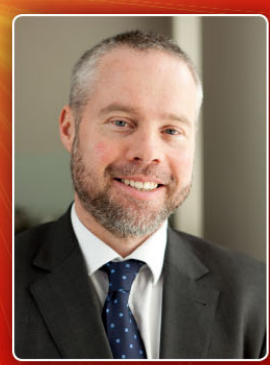




\title{
Self-excited coupled-microcantilevers for mass sensing
}

\author{
Daichi Endo, ${ }^{1}$ Hiroshi Yabuno, ${ }^{1, a)}$ Keiichi Higashino, ${ }^{2}$ Yasuyuki Yamamoto, ${ }^{3}$ \\ and Sohei Matsumoto ${ }^{4}$ \\ ${ }^{1}$ Graduate School of Systems and Information Engineering, University of Tsukuba, \\ Tsukuba Science City 305-8573, Japan \\ ${ }^{2}$ Graduate School of Science and Technology, Keio University, Yokohama, Kanagawa 223-8522, Japan \\ ${ }^{3}$ Material Properties Division, National Institute of Advanced Industrial Science and Technology (AIST), \\ Tsukuba Science City 305-8564, Japan \\ ${ }^{4}$ Hetero Convergence Team, National Institute of Advanced Industrial Science and Technology (AIST), \\ Tsukuba Science City 305-8564, Japan
}

(Received 3 April 2015; accepted 2 May 2015; published online 2 June 2015)

\begin{abstract}
This paper reports ultrasensitive mass detection based on the relative change in the amplitude ratio of the first mode oscillation using self-excited coupled microcantilevers. The method proposed and demonstrated using the macrocantilevers in the previous study can measure eigenstate shifts caused by objects with high accuracy without being affected by the viscous damping effect of measurement environments. In this study, moving towards the use of this method for small mass measurements, we established the self-excited coupled microcantilevers and we have achieved in measurements of very small mass (about $1 \mathrm{ng}$ ) with 1\% order of error. (C) 2015 AIP Publishing LLC.

[http://dx.doi.org/10.1063/1.4921082]
\end{abstract}

Recently, high-performance sensors using a microcantilever have been developed for a variety of purposes including surface sensing by atomic force microscope, ${ }^{1}$ measurements of substances such as $\mathrm{pH}$ in solution, ${ }^{2-4}$ measurements of viscosity in highly viscous liquid, ${ }^{5}$ and mass sensing by artificial nose. In particular, the use of cantilever in ultrasensitive mass sensors has been widely investigated. ${ }^{6-8}$ Many researches have employed the ways through the shift of the natural frequency of oscillators depending on measured additional mass. The natural frequency shift is usually detected from the frequency response curve under forced or external excitation. ${ }^{9-12}$ However, this method requires high accuracy measurement of the frequency shift in a vacuum because in viscous environments the peak of the frequency response curve is not clear and there is no peak for much more viscosity. In order to overcome these difficulties in the detection of the natural frequency, a method using self-excited oscillation produced through the velocity feedback of a cantilever has been utilized. ${ }^{1,13,14}$ On the other hand, it is known that the method based on the eigenstate shift in coupled cantilevers ${ }^{15}$ has better accuracy compared to based on the conventional natural frequency shift. ${ }^{16,17}$ The eigenstate has been identified from the magnitude ratio of the resonance peak of the frequency response curve in the coupled cantilevers under the external or forced excitation. ${ }^{16}$ However, because it relies on the frequency response curve, the method encounters the same issue as the method based on the frequency shift or may have low accuracy in the high-viscosity regime.

As a method to overcome such difficulties, we proposed a self excitation for coupled cantilevers. ${ }^{18}$ The method has the advantage that the coupled cantilevers respond with the eigenstate because self-excited oscillation occurs in the state where the viscous effect in the measurement environment is compensated by feedback force. Specifically, it is expected

${ }^{\text {a)} E l e c t r o n i c ~ m a i l: ~ y a b u n o @ e s y s . t s u k u b a . a c . j p ~}$ that the very small mass sensing can be realized not only with high-accuracy but also in high viscosity environments.

In this paper, we achieve using the micro-coupled cantilevers as Fig. 1 the method proposed in our previous study ${ }^{18}$ and actually measure very small mass. The accuracy of experimental results of the mass sensing is quantitatively discussed, and shows that it is possible to measure the mass with high accuracy without a special measurement environment, such as an ultrahigh vacuum to provide a high quality factor ( $Q$ factor).

As in the previous study, we introduce a discretized analytical model, ${ }^{16}$ as shown in Fig. 2, where $x_{1}$ and $x_{2}$ correspond the displacements of cantilevers, 1 and 2, relative to the excitation displacement $\Delta x$ corresponding to the lateral excitation of the supporting point of the coupled cantilevers, respectively. In Fig. 2, $m$ is the equivalent mass of cantilevers,

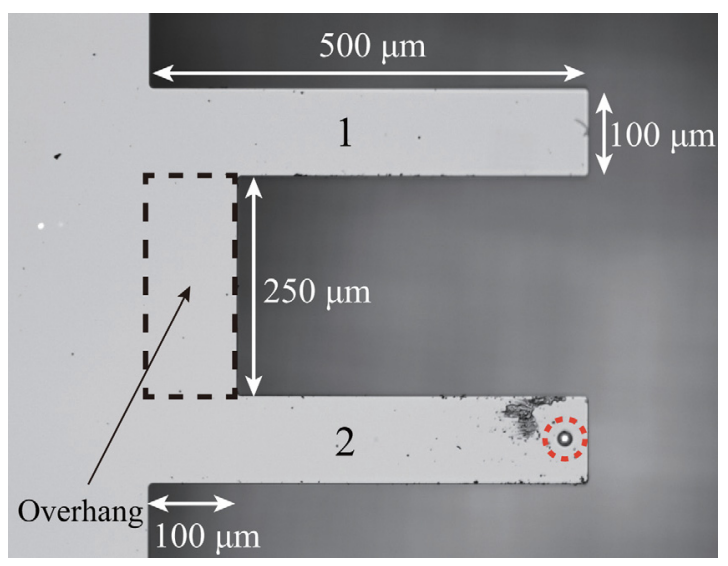

FIG. 1. Picture of the coupled microcantilevers fabricated by etching from an SOI wafer and a polystyrene microsphere to be measured on the tip of the cantilever 2 (surrounded by a red dashed circle). The area surrounded by a black dashed line corresponds to the overhang. The dimensions of each cantilever are $500 \mu \mathrm{m} \times 100 \mu \mathrm{m} \times 10 \mu \mathrm{m}$. The width and length of overhang are $250 \mu \mathrm{m}$ and $100 \mu \mathrm{m}$, respectively. 


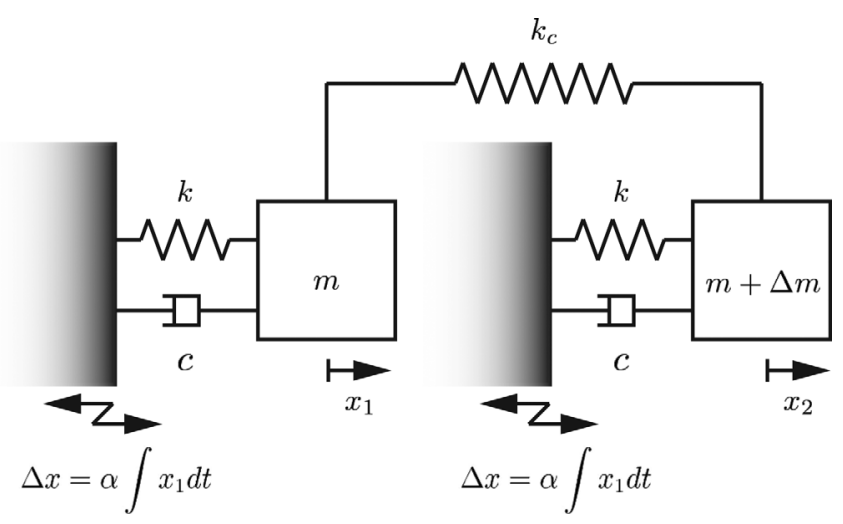

FIG. 2. A discretized analytical model for mass sensing. Each cantilever is modeled as a mass-spring-damping system, and the overhang coupling effect is described as the spring between identical two cantilevers.

1 and $2, \Delta m$ is the mass to be measured, $k$ and $c$ are, respectively, the equivalent stiffness and the viscous damping coefficient, and $k_{c}$ is the equivalent stiffness of the overhang coupling two cantilevers as shown in Fig. 1.

Here, in order to generate self-excited oscillation in coupled microcantilevers, we set $\Delta x$ as the integral feedback of the displacement $x_{1}$ of cantilever 1 as follows:

$$
\Delta x=\alpha \int x_{1} d t
$$

where $\alpha$ is the feedback gain. Then, we derive the dimensionless equation of motion. The dimensionless equation of motion of the coupled cantilevers is expressed in matrix form as follows:

$$
\begin{aligned}
& {\left[\begin{array}{l}
\ddot{x}_{1} \\
\ddot{x}_{2}
\end{array}\right]+\left[\begin{array}{cc}
2 \gamma+\beta & 0 \\
\beta & 2 \gamma /(1+\delta)
\end{array}\right]\left[\begin{array}{l}
\dot{x}_{1} \\
\dot{x}_{2}
\end{array}\right]} \\
& +\left[\begin{array}{cc}
1+\kappa & -\kappa \\
-\kappa /(1+\delta) & (1+\kappa) /(1+\delta)
\end{array}\right]\left[\begin{array}{l}
x_{1} \\
x_{2}
\end{array}\right]=\left[\begin{array}{l}
0 \\
0
\end{array}\right],
\end{aligned}
$$

where the dot indicates the derivative of dimensionless time $t^{*}=\sqrt{k / m} t$, and $\kappa, \delta, \beta$, and $\gamma$ are dimensionless parameters as expressed as follows:

$$
\kappa=\frac{k_{c}}{k}, \delta=\frac{\Delta m}{m}, \beta=\alpha \sqrt{\frac{m}{k}}, \gamma=\frac{c}{2 \sqrt{m k}} .
$$

The parameters, $\kappa$ and $\delta$, are the dimensionless coupling stiffness and dimensionless additional mass ratio, respectively, and $\beta$ and $\gamma$ are the dimensionless feedback gain and the dimensionless viscous damping coefficient, respectively. The first eigenstate of coupled microcantilevers is expressed as follows:

$$
p_{1}=\left[1 /\left\{\sqrt{(1+\delta)+\frac{\delta^{2}}{4 \kappa^{2}}\left(1+\kappa^{2}\right)}-\frac{\delta}{2 \kappa}(1+\kappa)\right\}\right] .
$$
follows:

$$
\beta<-2 \gamma-\frac{\gamma\left(\omega_{2}^{2}-\omega_{1}^{2}\right)^{2} \omega_{1}^{2} \delta}{\left\{8 \gamma^{2} \omega_{1}^{4}+2 \omega_{1}^{2}\left(\omega_{2}^{2}-\omega_{1}^{2}\right)^{2}\right\} \kappa},
$$

where $\omega_{1}$ and $\omega_{2}$ are eigenvalues corresponding to the dimensionless first and second modes, respectively, selfexcited oscillation can be produced in the coupled macrocantilevers with only the first mode of the coupled cantilevers (in-phase mode). ${ }^{18}$

Figure 3 is the experimental setup of mass sensor with a self-excited coupled microcantilevers, and Fig. 4 is the optical path of the experiment setup and the block diagram. The system consists of a XY-axis-stage, a piezoelectric actuator, a deflecting prism, a reflecting mirror, and laser Doppler vibrometers. Coupled microcantilevers are placed on the $\mathrm{XY}$-axis-stage and fixed by a leaf spring. The piezoelectric actuator (FUJI CERAMIC Corp.: Z1T5x5S-SYXN(C-82)) provides the displacement excitation to the support point $(=\Delta x)$, so that cantilevers, 1 and 2 , connected through the overhang are moved simultaneously according to the proposed feedback rule. The vibrations of cantilevers are measured by laser Doppler vibrometers (ONO SOKKI.: LV-1610), and each laser beam is made so as not to interfere with each other by the reflecting mirror and the deflecting prism as shown Fig. 4. The laser beam for measuring cantilever 1 passes through the prism, while the laser beam for measuring the cantilever 2 is changed in direction by $90^{\circ}$ by the deflecting prism. Each laser beam is reflected by the surface of cantilevers and returns to the laser Doppler vibrometers. In order to induce self-excited oscillation in the coupled microcantilevers, the signal of the displacement of cantilever 1 measured by the laser Doppler vibrometer is integrated by an analog integrator and amplified by a feedback gain $\alpha$. Furthermore, based on an amplitude control method previously proposed for a self-excited single cantilever, ${ }^{1}$ we applied a nonlinear feedback described as $\alpha^{\prime} \int x_{1}^{3} d t$ not to grow without boundary with time, but to reach the steady state with a small constant amplitude; the theoretical validity of the nonlinear feedback control for the coupled cantilevers will be reported in another paper. By making the amplitude of the self-excited cantilevers small, the nonlinear effect due to the large deflection does not occur. As a result, we can oscillate the coupled cantilevers with the desired linear mode. The linear feedback

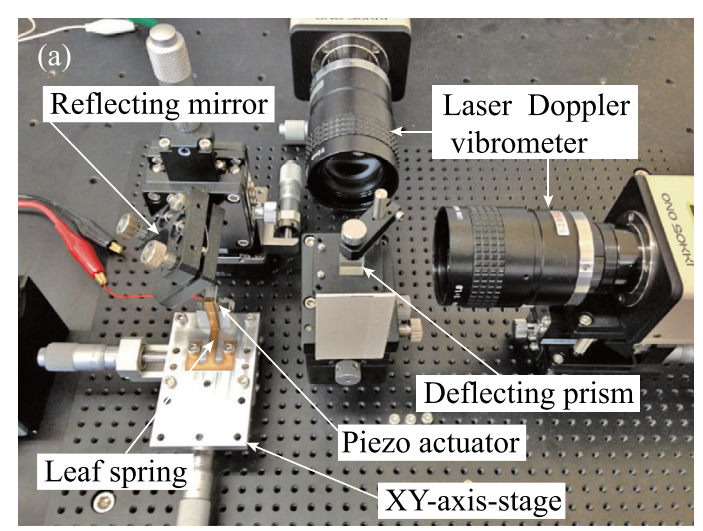

FIG. 3. Top view of the mass sensing system. Coupled microcantilevers are placed on the XY-axis-stage and moved by the piezoelectric actuator according to the proposed feedback control to produce self-excited oscillation. 


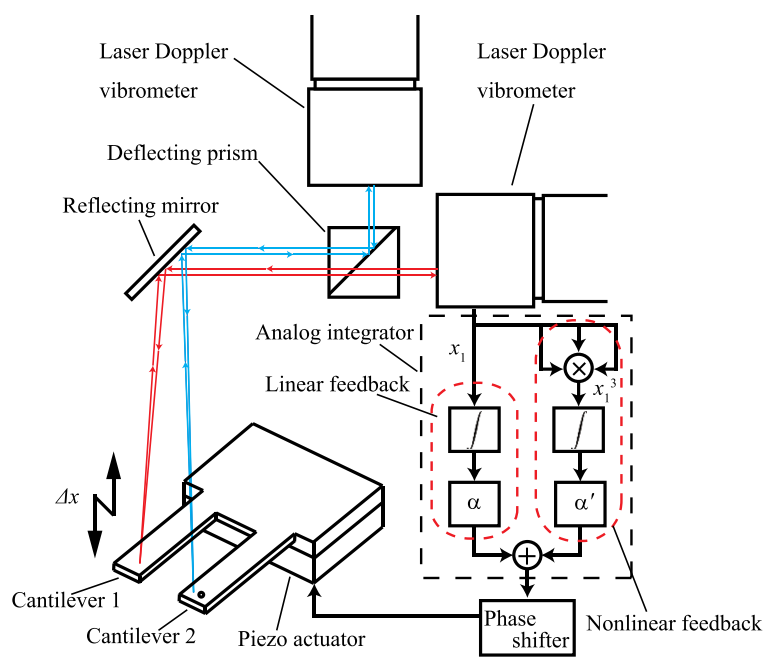

FIG. 4. Optical path in the experimental setup and block diagram. The laser beams to measure the vibrations of cantilevers, 1 and 2, are made so as not to interfere with each other by the reflecting mirror and the deflecting prism. The linear feedback produces self-excited oscillation in the coupled microcantilevers, and the nonlinear feedback makes the amplitude of the selfexcited cantilevers small.

signal and the nonlinear feedback signal are added in the ana$\log$ integrator. The phase of the self-excited frequency component in the output is adjusted to delay just $\pi / 2 \mathrm{rad}$ from that of $x_{1}$ by the phase-shifter and the output of the phase shifter is given to the piezoelectric actuator. Then, selfexcited oscillation with constant amplitude was realized in the coupled microcantilevers.

Before starting the experiment with self-excited oscillation, we clarified the coupling characteristic of the fabricated mass sensor. The dimensionless coupling stiffness is experimentally identified as $\kappa=\frac{1}{2}\left\{\left(f_{2} / f_{1}\right)^{2}-1\right\} \simeq 1.44 \times 10^{-2}$ based on the relationship between the first and second natural frequencies, $f_{1}=55.3 \mathrm{kHz}$ and $f_{2}=56.1 \mathrm{kHz}$. The equivalent stiffness $k$ is experimentally identified as $k=3 E I / l^{3}$ $=37 \mathrm{~N} / \mathrm{m},{ }^{19}$ by using the value of $E=185 \mathrm{GPa}$ as Young's modulus of silicon, the value of $I=8.33 \mu \mathrm{m}^{4}$ as the moment of inertia of area calculated with the dimensions of a cantilever, and the value of $l=500 \mu \mathrm{m}$ as the length of a cantilever in Fig. 1. The equivalent mass in the discrete model corresponding to the mass of the cantilever is derived as $m=k /\left(2 \pi f_{1}\right)^{2} \simeq 306 \mathrm{ng}$.

We generated self-excited oscillation to the coupled microcantilevers by increasing the absolute value of the negative feedback gain $\alpha$ gradually. Exceeding the value at which the feedback gain is critical, i.e., $\beta$ equals right-hand side of Eq. (5) in theory, self-excited oscillation was induced to the coupled microcantilevers. The spectrum analysis of the selfexcited cantilevers in air is obtained from the spectrum analyzer (Agilent Technologies.: EXA signal analyzer N9010A) as shown in Fig. 5, where the ordinate and abscissa indicate the voltage corresponding to the magnitude of the amplitude and the response frequency, respectively. According to Fig. 5, the cantilevers are vibrated at 1:1 amplitude ratio.

Next, we placed a polystyrene microsphere (Polysciences, Inc.: Polybead) on the tip of the cantilever 2 as shown in Fig. 1. The mean diameter is $15.0 \mu \mathrm{m}$ and the ratio of standard deviation to the mean diameter ( $\mathrm{CV}$ value) is $10 \%$ or less. Its

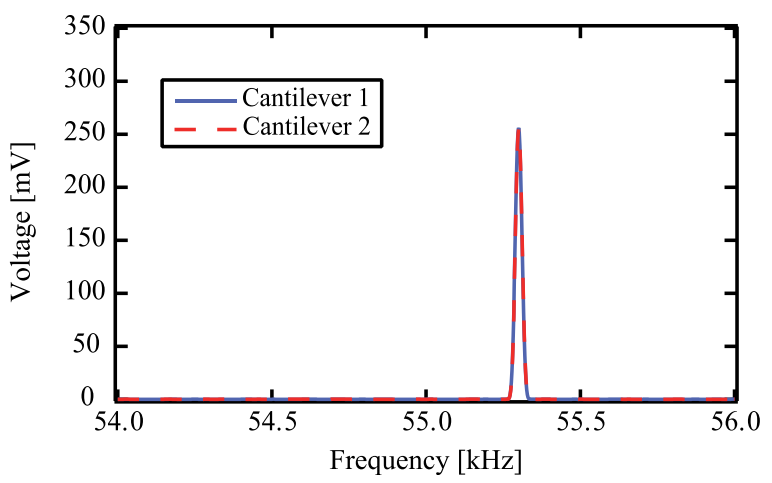

FIG. 5. Spectrum analysis of the self-excited cantilevers in air under the feedback control without the mass to be measured; blue solid line and red dashed line correspond to the spectrum of cantilevers 1 and 2, respectively. The ordinate indicates the voltage corresponding to the magnitude of the amplitude and the abscissa indicates frequency. In the experiment, we adjusted the measurement point of cantilever 2 so that the ratio of the amplitude between cantilever 1 and 2 were 1:1.

mass is $1855 \mathrm{pg}$ calculated from the density of the polystyrene $\left(=1.05 \mathrm{~g} / \mathrm{cm}^{3}\right)$ and the volume of a sphere with the mean diameter. Note that the ratio of the mass to be measured to the equivalent mass $306 \mathrm{ng}$ of the cantilever is $6.06 \times 10^{-3}$. The spectrum analysis of the self-excited cantilevers in air, with one microsphere placed on the cantilever 2 is obtained from the spectrum analyzer as shown in Fig. 6 . The amplitude ratio of cantilever 2 to cantilever 1 is 1.24 , which means the mass ratio $6.06 \times 10^{-3}$ induced an eigenstate shift of 0.24 , i.e., the relative change in the eigenstate is $24 \%$.

In addition, we attached a plurality of polystyrene microspheres, and measured the response amplitude ratios in the self-excited coupled microcantilevers. Figure 7 shows the relationship between the experimentally determined mass values of the microsphere $\Delta m_{e}$ and their nominal values $\Delta m$ calculated from the dimensions and density of the microsphere. Since the CV value in the nominal dimensions of the microsphere is $10 \%$ or less, the standard deviation of nominal values of the mass is shown as error bars. As can be seen from Fig. 7, the experimentally determined mass values are almost consistent with a straight line of gradient 1 , and the error between the nominal and experimental values is $4.9 \%$ at maximum. The sensing accuracy of the coupled

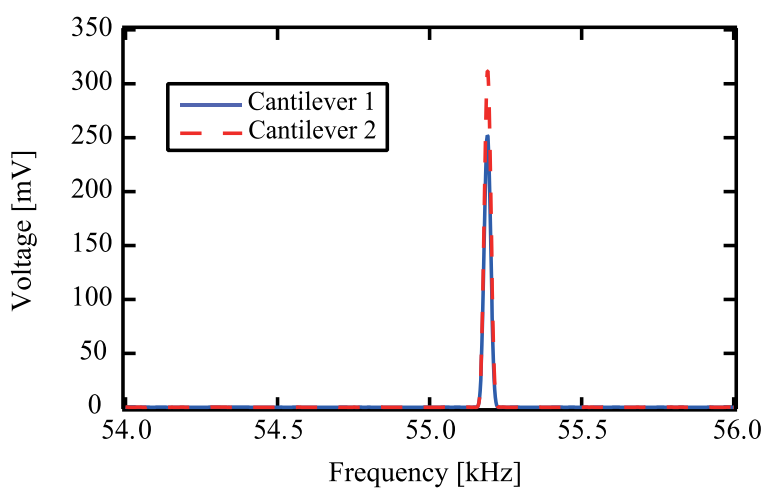

FIG. 6. Spectrum analysis of the self-excited cantilevers in air under the feedback control with a mass of $1855 \mathrm{pg}$ added to cantilever 2; blue solid line and red dashed line correspond to the spectrum of cantilevers 1 and 2 , respectively. The mass ratio, $6.06 \times 10^{-3}$, induces the eigenstate shift and changes the amplitude ratio between cantilevers 1 and 2 from $1: 1$ to $1: 1.24$. 


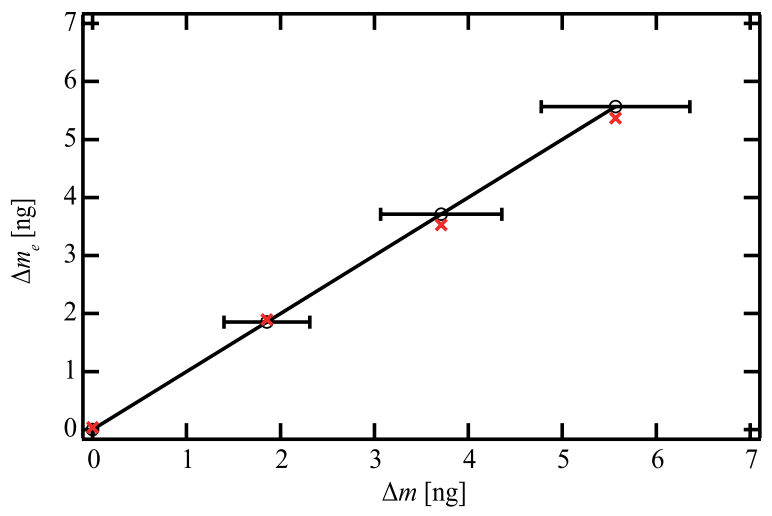

FIG. 7. Measurement results for microspheres. The abscissa and ordinate indicate, respectively, the nominal and experimental values of the mass of the microsphere. In the figure, $\bigcirc$ and error bars are nominal values of the mass calculated from the dimensions of the microsphere and the standard deviation of nominal values of the mass, respectively, and $\times$ is mass values obtained from the eigenstates shift under self-excited oscillation in the experiment. When $\times$ falls on the straight line of gradient 1 , the experimentally determined mass value is in complete agreement with the nominal value.

cantilevers may be affected by the position of the mass to be measured. It is theoretically clarified that the error of measurement value is proportional to the ratio of the deviation of the position from the free end of the cantilever to the cantilever length; the details will be mentioned in another paper due to page limitation. In the experiments, the deviation from the free end of the cantilever is at largest in the order of the diameter of a microsphere. Thus, the ratio is in order of $O(0.01)$ and the deviation of the mass sensing in the experiments can be neglected. Therefore, we can say, that the mass sensor utilizing self-excited oscillation can measure small objects with such a high accuracy in air. In other words, we need not prepare the special measurement environment, such as an ultrahigh vacuum to provide a high $\mathrm{Q}$ factor in the frequency response curve. Spletzer et al. invented the ultrasensitive mass sensor using coupled microcantilevers under external or forced excitation; however, the measurement accuracy was not discussed in their study. ${ }^{16}$ By performing a comparison of the experimental and theoretical values, we have assessed the measurement accuracy in this study.

In conclusion, we have achieved a self-excited type mass sensor using micro-coupled cantilevers. The fabricated mass sensor can measure 1855 pg with high accuracy. Unlike the conventional method, which uses the response frequency under external or forced excitation, the fabricated mass sensor based on self-excited oscillation can detect the object to be measured with high accuracy without a special measurement environment to, for example, increase the $\mathrm{Q}$ factor. This is because the utilization of self-excitation can avoid the effect of the viscous damping effect in the measurement environment. In the near future, we will achieve the very small mass measurement in liquid using the self-excited coupled microcantilevers.

The authors would like to thank Dr. T. Yamamoto of The National Institute of Advanced Industrial Science and Technology (AIST) for helpful discussion about fabricating cantilevers. This work was partially supported by a Grant-inAid for Scientific Research C (Grant No. 25420195) from the Japan Society for the Promotion of Science (JSPS).

${ }^{1}$ H. Yabuno, H. Kaneko, M. Kuroda, and T. Kobayashi, Nonlinear Dyn. 54, 137 (2008).

${ }^{2}$ R. Bashir, J. Z. Hilt, O. E. Gupta, and N. A. Peppas, Appl. Phys. Lett. 81, 3091 (2002).

${ }^{3}$ R. Raiteri, H. J. Butt, and M. Grattarola, Electrochim. Acta 46, 157 (2000).

${ }^{4}$ J. Fritz, M. K. Baller, H. P. Lang, H. Rothuizen, P. Vettiger, E. Meyer, H. J. Guntherodt, C. Gerber, and J. K. Gimzewski, Science 288, 316 (2000).

${ }^{5}$ H. Yabuno, K. Higashino, M. Kuroda, and Y. Yamamoto, J. Appl. Phys. 116, 124305 (2014).

${ }^{6}$ K. L. Ekinci, X. M. H. Huang, and M. L. Roukes, Appl. Phys. Lett. 84, 4469 (2004).

${ }^{7}$ T. Ono, X. Li, H. Miyashita, and M. Esashi, Rev. Sci. Instrum. 74, 1240 (2003).

${ }^{8}$ E. G. Santos, D. Ramos, J. Martinez, M. F. Regulez, R. Garcia, A. S. Paulo, M. Calleja, and J. Tamayo, Nat. Nanotechnol. 5, 641 (2010).

${ }^{9}$ G. Y. Chen, T. Thundat, E. A. Wachter, and R. J. Warmack, J. Appl. Phys. 77, 3618 (1995).

${ }^{10}$ B. Ilic, D. Czaplewski, M. Zalalutdinov, H. G. Craighead, P. Neuzil, C. Campagnolo, and C. Batt, J. Vac. Sci. Technol., B 19, 2825 (2001).

${ }^{11}$ H. Sone, Y. Fujinuma, and S. Hosaka, Jpn. J. Appl. Phys., Part 1 43, 3648 (2004).

${ }^{12}$ Z. J. Davis and A. Boisen, Appl. Phys. Lett. 87, 013102 (2005).

${ }^{13}$ Y. Lee, G. Lim, and W. Moon, Sens. Actuators, A 130, 105 (2006).

${ }^{14}$ T. Okajima, H. Sekiguchi, H. Arakawa, and A. Ikai, Appl. Surf. Sci. 210, 68 (2003).

${ }^{15}$ M. Sato, B. E. Hubbard, A. J. Sievers, B. Ilic, D. A. Czaplewski, and H. G. Craighead, Phys. Rev. Lett. 90, 044102 (2003).

${ }^{16}$ M. Spletzer, A. Raman, A. Q. Wu, X. Xu, and R. Reifenberger, Appl. Phys. Lett. 88, 254102 (2006).

${ }^{17}$ M. Spletzer, A. Raman, H. Sumali, and J. P. Sullivan, Appl. Phys. Lett. 92, 114102 (2008).

${ }^{18}$ H. Yabuno, Y. Seo, and M. Kuroda, Appl. Phys. Lett. 103, 063104 (2013).

${ }^{19}$ S. Timoshenko and D. H. Young, Elements of Strength of Materials, 5th ed. (D. Van Nostrand Company, Inc., 1968), Chap. 8, p. 212. 